\title{
The Production and Destruction of Negative Ions
}

Progress Report and Future Plans

David J. Pegg

Department of Physics, University of Tennessee,Knoxville, TN

September 1, 1992-August 31, 1993

Prepored for the US DOE under grant no: DOE-FG05-85ER13456

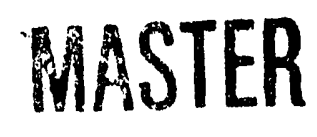

OISTRIBUTIDN OF THIS DOCUMENT IS UALIMITEN 


\section{Table of Contents}

A. Introduction 1

B. Photodetachment of B- 2

C. Photodetachment of Be-

a. Introduction 2

b. Electron Affinity $\quad 4$

c. Photoelectron Angular Distribution 8

d. Photodetachment Cross Section 8

D. Future Plans 9

$\begin{array}{ll}\text { E. References } & 11\end{array}$

F. List of Publications: 1992-93 12 


\section{A.Introduction}

During the present grant period we continue our study of the process of single photon absorption-single electron detachment from fewelectron atomic negative ions. A crossed beam apparatus is being used to perform energy- and angle-resolved photoelectron spectroscopic measurements following photodetachment. The apparatus and the experimental techniques used have been described in detail by Pegg [1]. Those electrons ejected in the direction of motion of the ion beam i.e. forward-directed electrons are collected and energy analyzed. The kinetic energies and yields of the photoelectrons are obtained by fitting the spectral peaks to Gaussian functions. Electron affinities, asymmetry parameters and cross sections are determined from these measurements. We have circumvented the difficult task of making an absolute measurement of a cross section by using a ratio method in which the cross section for the ion of interest is measured relative to that of a reference ion. Our primary reference is $\mathrm{D}^{-}\left(\mathrm{H}^{-}\right)$whose photodetachment cross section is known from theory to better than $3 \%$. A secondary reference is $\mathrm{Li}^{-}$whose cross section is known from theory to about $10 \%$. Our previous measurement of the $\sigma\left(\mathrm{Li}^{-}\right) / \sigma\left(\mathrm{D}^{-}\right)$ ratio allows us to indirectly link measurements made using the $\mathrm{Li}^{-}$ secondary reference to the $D^{-}$primary reference. To avoid measuring the relative efficiency for collecting and detecting electrons from the ion beam of interest and the reference beam, we carefully choose the ion beam energies in such a way that the energies of the two groups of detached electrons are kinematically "amplified" to the same energy in the laboratory frame. This procedure, which was developed in our laboratory, considerably enhances the precision of the ratio measurement.

During this grant period we finished our study of the photodetachment of $\mathrm{Li}^{-}$and this data was used as the subject matter of the Ph.D thesis of Joseph Dellwo [2]. We have begun to investigate the photodetachment of the stable ion, $\mathrm{B}^{-}$and the metastable ion, $\mathrm{Be}^{-}$ There exists no previous information, either experimental or 
theoretical, on the phntodetachment cross sections and photoelectron asymmetry parameters for these two ions.

\section{B. Photodetachment of B-}

The $B^{-}$ion is stable. The only bound state is the $\left(1 s^{2} 2 s^{2} 2 p^{2}\right)^{3 p}$ state. We have made a series of measurements of the cross section ratio, $\sigma\left(\mathrm{B}^{-}\right) / \sigma\left(\mathrm{Li}^{-}\right)$, at a photon energy of $2.075 \mathrm{eV}$. At this photon energy photodetachment can only proceed via the ${ }^{2} \mathrm{P} \varepsilon s, d$ channels i.e. hv $+B^{-}$ $\left(2^{3} P\right) \rightarrow B\left(2^{2} P\right)+e^{-}(\varepsilon s, d)$. The angular distribution of the photoelectrons detached in this process has been previously studied by our group. The asymmetry parameters were measured at several photon energies in the visible and the results can be found in the paper by Liu et al. [3]. The measured ratio, $\sigma\left(\mathrm{B}^{-}\right) / \sigma\left(\mathrm{Li}^{-}\right)$, is $0.32 \pm 0.04$. This result can be normalized to our previous measurement of $\sigma\left(\mathrm{Li}^{-}\right)$ $=63.5 \pm 5.7 \mathrm{Mb}$ to yield a preliminary result of $\sigma\left(\mathrm{B}^{-}\right):=20 \pm 3 \mathrm{Mb}$.

\section{Photodetachment of Be-}

\section{(a) Introduction}

The $\mathrm{Be}$ - ion belongs to an interesting class of negative ions that are metastable against autodetachment. This anion is formed in the $\left(1 s^{2} 2 s^{2} p^{2}\right)^{4} p$ state when an electron attaches itself to the metastable (1 $\left.s^{2} 2 s 2 p\right)^{3} p$ state in Be (see Fig. 1). At a photon energy of $2.075 \mathrm{eV}$ photodetachment of $\mathrm{Be}^{-}$proceeds via the $3 \mathrm{PEs}_{\mathrm{s}} \mathrm{d}$ channels. We have initiated a program to measure the electron affinity of $\mathrm{Be}\left({ }^{3} \mathrm{P}\right)$ and the cross sections for detaching an electron from $\mathrm{Be}^{-}$at various photon energies in the visible. The measurements are somewhat difficult and time consuming due to the low intensity of the ion beams that we extract from the ion source. We feel the effort to be worthwhile, however, since there have been no previous measurements of the electron affinity of Be accurate enough to meaningfully test theoretical predictions. An even more important justification is the fact that there have been no previous investigations, either experimental or theoretical, of the cross 


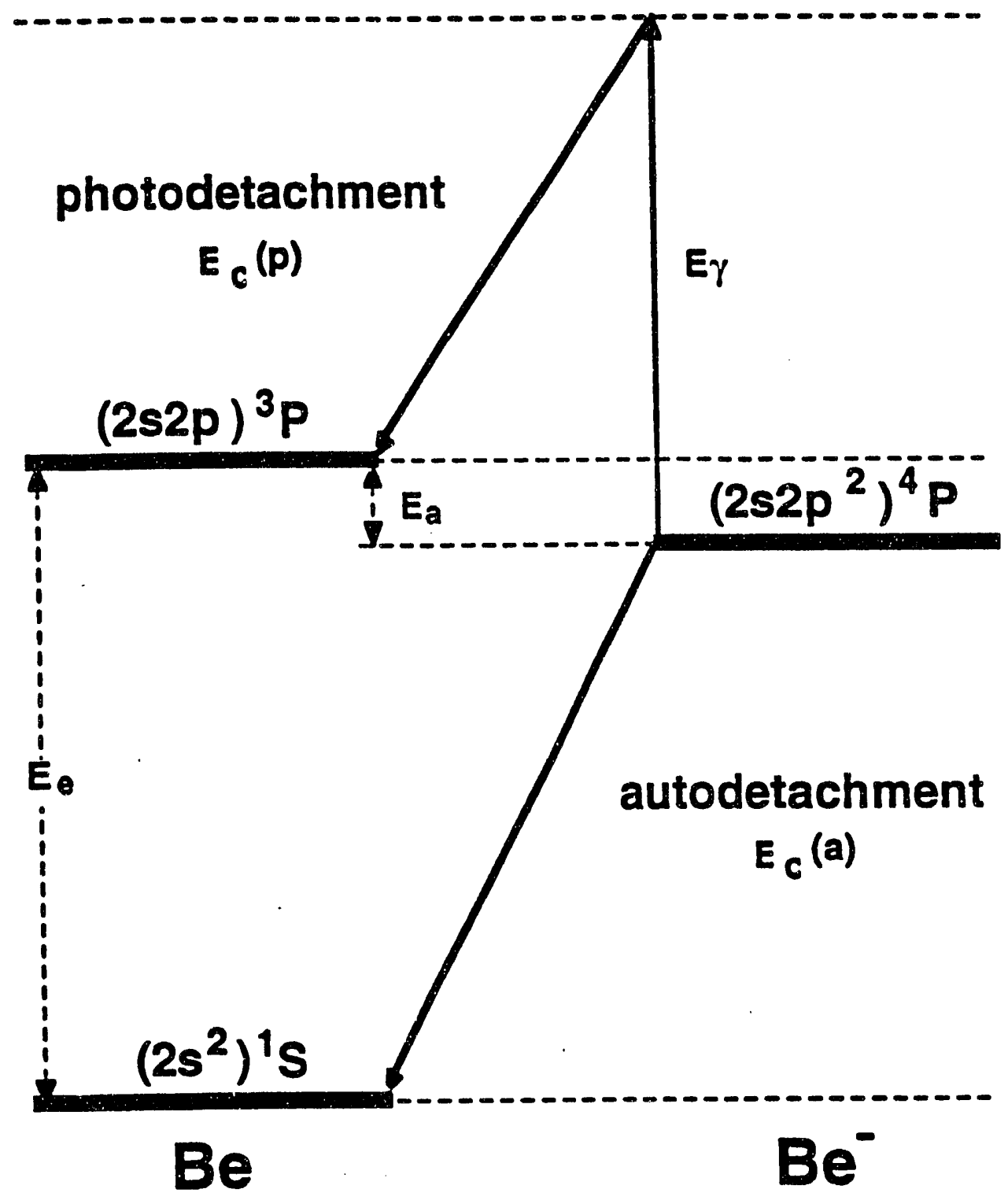

Figure 1 
section for photodetachment of this ion and the angular distributions of the detached photoelectrons.

\section{(b) Electron Affinity}

We have used the technique of Photodetached Electron Spectroscopy $(P E S)$ to determine the electron affinity of $\mathrm{Be}\left({ }^{3 P}\right)$. This measurement has been completed in the current grant period. The objective was to measure the energy of the detached electrons, $E_{c}(p)$, for a known value of the photon energy, $E_{\gamma}$. The electron affinity, $E_{a}$, can then be determined from the energy balance equation, in this case, $E_{a}=E_{\gamma}$ $E_{c}(p)$ ( see Fig. 1). The ion-frame energy of the ejected electron, $E_{c}$, is related to the measured laboratory-frame energy, $E_{L}$, for the present case of forward-directed electron spectroscopy, via the kinematic transformation equation: $\sqrt{ } E_{L}=\sqrt{ } E_{c}+\sqrt{ } \varepsilon$. The quantity $\varepsilon$ represents the ion beam energy reduced by the electron-to-ion mass ratio. In the present work we were able to determine the $\mathrm{Be}^{-}$ion beam energy to better than $1 \%$ using an in-situ analysis of the two peaks in the $\mathrm{He}^{-}$reference spectrum which was obtained at the same ion beam energy and under the same experimental conditions (see Fig.2a). These two peaks were also used to calibrate the energy scale of the $\mathrm{Be}^{-}$spectrum (see Fig.2b). Checks on the calibration were also made by use of the peaks associated with the photodetachment of electrons from $\mathrm{B}^{-}$and $\mathrm{Li}$ - beams.

Table 1. The Electron Affinity (EA) of $\mathrm{Be}\left(1 s^{2} 2 s 2 p\right)^{3} \mathrm{P}$

$\mathrm{EA}(\mathrm{meV})$

$240 \pm 100$

285

$217.7 \pm 57.1$

$195 \pm 90$

$276.1 \pm 6.5$

$261 \pm 10$
Method

Theoretical

Theoretical

Theoretical

AES

Theoretical

PES
Reference

[4]

[5]

[6]

[7]

[8]

$261 \pm 10 \quad$ PES $\quad$ This work




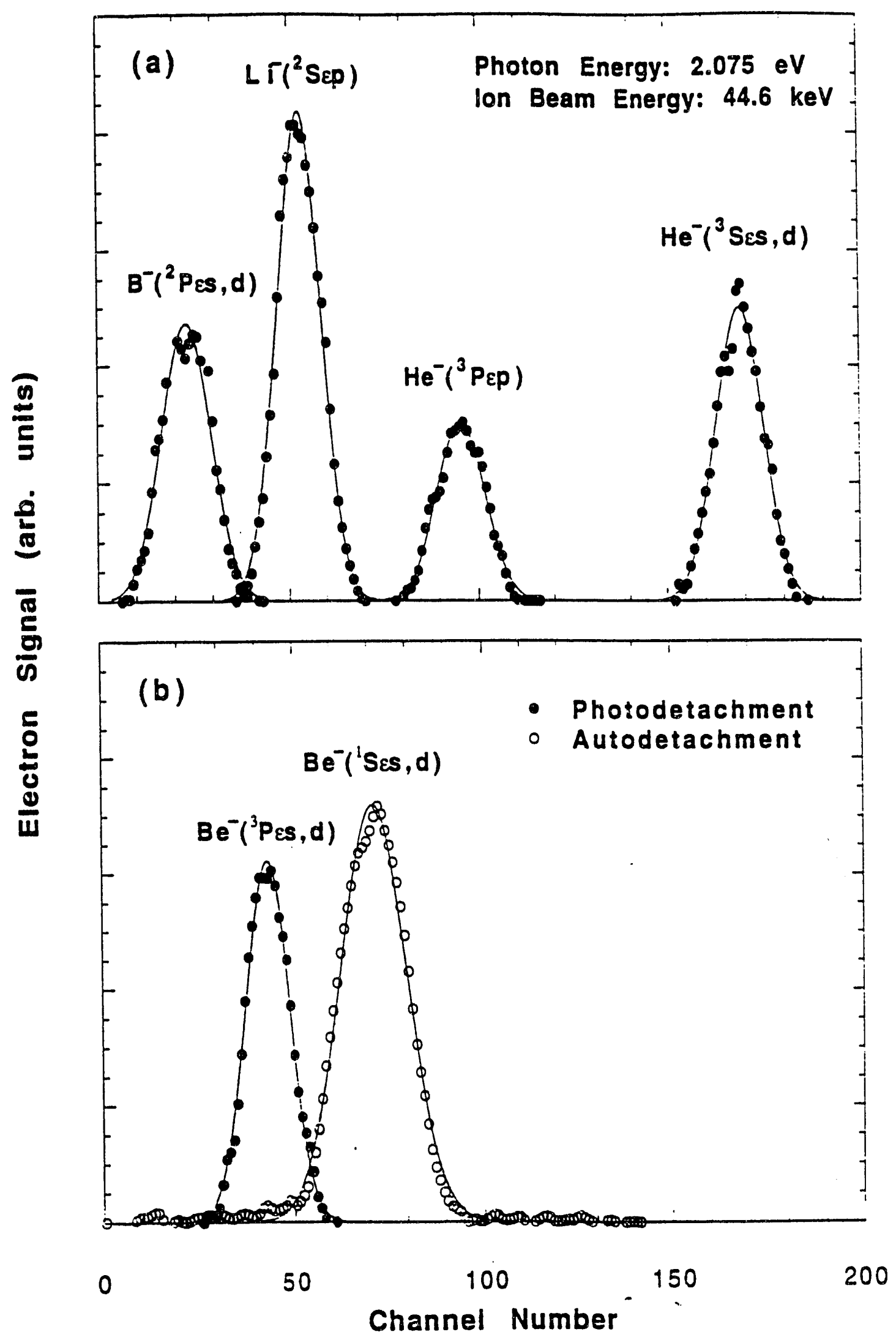

Figure 2 
The result of the PES measurement was $E_{c}(p)=1.814 \pm 0.010 \mathrm{eV}$. This electron energy corresponds to an electron affinity of $E_{a}(23 P)=$ $261 \pm 10 \mathrm{meV}$. The present result, obtained by PES, is compared in Table 1 with a previous measurement obtained by Autodetached Electron Spectroscopy (AES) and theoretical estimates. The PES result is sufficiently accurate to meaningfully test, for the first time, the accurate calculation of Bunge [8]. The measured value is seen to be somewhat lower than the currently accepted theoretical value although there is agreement within the overlapping error limits.

Since the $\mathrm{Be}-$ ion in the $2^{4} \mathrm{P}$ state may autodetach via the $2^{1} \mathrm{~S} \varepsilon s, \mathrm{~d}$ channels, one can perform an additional consistency check by measuring the energy of the autodetached electrons, $E_{c}(a)$. This has been achieved in the present work by the use of a hybrid method in which the spectrum of autodetached electrons was accumulated using AES and the ion beam energy and energy scale calibration were determined from the PES of $\mathrm{He}^{-}$. The peak associated with the autodetachment of $\mathrm{Be}^{-}$is shown to the right in Fig. $2 \mathrm{~b}$ and the $\mathrm{He}^{-}$ reference spectrum is shown to the right of Fig. 2a. The result of this APES measurement was $E_{c}(a)=2.463 \pm 0.021 \mathrm{eV}$. Subtraction of this result from the accepted value of the excitation energy, $E_{e}$, of the 23P state of Be (see Fig.1) yields another value for the electron affinity of Be. If one assumes the measured photon spectroscopic value of $E_{e}=2.7252 \mathrm{eV}$ [9], one obtains a $B e\left(2^{3} \mathrm{P}\right)$ electron affinity of $E_{a}=262 \pm 21 \mathrm{meV}$. Alternatively, by combining the measured autodetached electron energy with the electron affinity of $E_{a}=0.261 \pm 0.010 \mathrm{eV}$ obtained from the PES measurement allows us to determine the $2^{1} \mathrm{~S}-2^{3 \mathrm{P}}$ energy separation in the Be atom. The present result of $E_{e}=2.723 \pm 0.007 \mathrm{eV}$ is in excellent agreement with the accepted value of $2.7252 \mathrm{eV}$ obtained from a difficult spectroscopic measurement using the rather weak intercombination line [10]. Although less accurate, the measurement does represents the first independent check of the excitation energy of the $\mathrm{Be}\left(2^{3} \mathrm{P}\right)$ state. Interestingly, the uncertainty limit on the sum of the two measured 


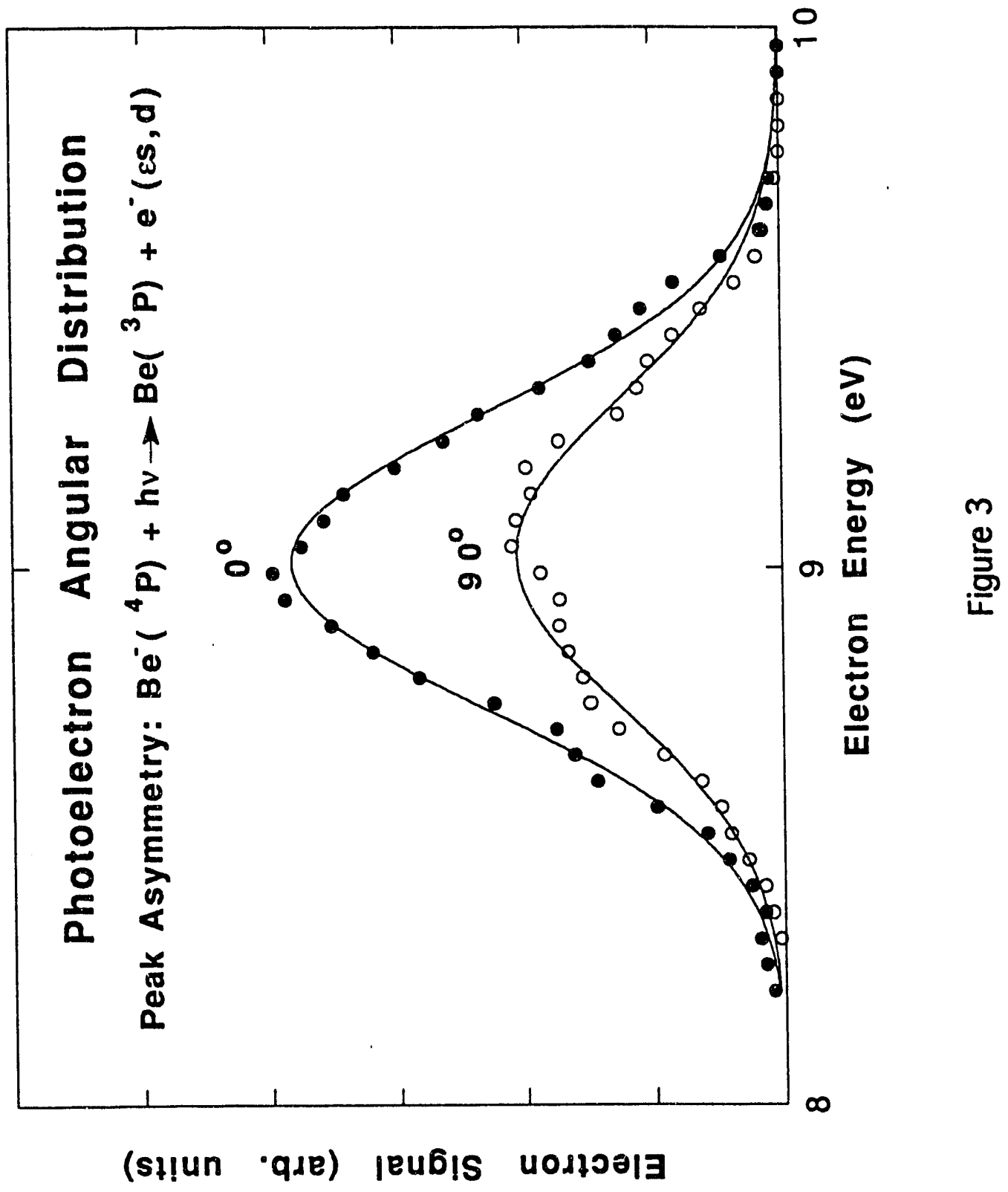


energies is considerably less than on each individual energy because of cancellations in the error propagation.

\section{(c) Photoelectron Angular Distribution}

We have made the first investigation of the angular distribution of electrons photodetached from a beam of metastable $\mathrm{Be}$ - ions. In the angle-dependent yield measurements the electron collection direction was held fixed in the forward direction while the electric vector of the linearly polarized laser beam was rotated using a double Fresnel rhomb. The angle scale of the apparatus was calibrated using the known angular distribution of electrons accompanying the photodetachment of a reference beam of $\mathrm{Li}$ - ions. Fig. 3 shows how the yield of photoelectrons changes when the polarization vector is directed parallel $\left(0^{\circ}\right)$ and perpendicular $\left(90^{\circ}\right)$ to the collection direction. The photoelectron angular distribution is characterized by a quantity called the asymmetry parameter which has been measured to be $0.49 \pm 0.02$ at a photon energy of $2.075 \mathrm{eV}$.

\section{(d) Photodetachment Cross Section}

We report a preliminary value for the cross section for photodetaching an electron from $\mathrm{Be}^{-}$at a photon energy of $2.075 \mathrm{eV}$. The experiment involved measuring the ratio $\sigma\left(\mathrm{Be}^{-}\right) / \sigma\left(\mathrm{D}^{-}\right)$and normalizing the result to a theoretical value of $\sigma\left(D^{-}\right)$. The ratio was found to be $0.90 \pm 0.09$ which corresponds to a value of $\sigma\left(\mathrm{Be}^{-}\right)=31 \pm 4$ $\mathrm{Mb}$. This result is more than a factor of three higher than the only previous measurement by Bae and Peterson [11] who quote a value of $9 \mathrm{Mb}$ at the approximately the same photon energy. The uncertainty on this measurement, which monitored neutral atom production, is estimated to be about $50 \%$. Measurements are continuing to verify this apparently large discrepancy. Unfortunately, to date, there have been no calculations of this quantity.

\section{DISCLAIMER}

This report was prepared as an account of work sponsored by an agency of the United States Government. Neither the United States Government nor any agency thereof, nor any of their employees, makes any warranty, express or implied, or assumes any legal liability or responsibility for the accuracy, completeness, or usefulness of any information, apparatus, product, or process disclosed, or represents that its use would not infringe privately owned rights. Reference herein to any specific commercial product, process, or service by trade name, trademark, manufacturer, or otherwise does not necessarily constitute or imply its endorsement, recommendation, or favoring by the United States Government or any agency thereof. The views and opinions of authors expressed herein do not necessarily state or reflect those of the United States Government or any agency thereof. 


\section{Future Plans}

During the 1993-94 grant period we propose to continue our photodetachment measurements on the few-electron $B e$ and $B$ anions at different photon energies in the visible in order to understand the spectral dependence of cross sections and asymmetry parameters. It is hoped that the work will stimulate theoretical activity in these areas.

We would also like to attempt some exploratory experiments during this time period. For example, the process of radiative autodetachment has been predicted theoretically by Nicolaides and his collaborators [11] but, thus far, it has not been observed. We plan to search for evidence of this process by studying the autodetachment of the simple three-electron $\mathrm{He}$ - ion. In the process of radiative autodetachment the de-excitation energy of the metastable $\mathrm{He}^{-}$ion is shared between an electron and a photon. A spectrum of autodetached electrons exhibits a peak corresponding to "pure" autodetachment i.e. the electron carries away all the excitation energy. A signature of radiative autodetachment would be the presence of a continuum in the electron spectrum on the low energy side of the peak resulting from a photon carrying away some of the energy.

Another exploratory experiment would be to investigate the production, by the charge exchange method, of the metastable $\left(2 p^{4}\right)^{1} S, 1 D$ states of $N$ - These states are estimated to have lifetimes against autodetachment of tens of microseconds. We have attempted, without success, to produce them by charge exchange when a beam of $\mathrm{N}^{+}$passes through a $\mathrm{Li}$ vapor target. In these collision partners the energy defect in the first collision preferentially produces the $N$ atom in it's $4 S$ ground state and not the metastable $2 D$ and $2 P$ states which are the parent states of the $1 D$ and $1 S$ states of $N$, respectively. Based on energy defects, Xe would be the best choice 
for a charge exchange gas in the first collision to optimize the production of merastable $\mathrm{N}$ atoms. Our plan would be to try to produce metastable $\mathrm{N}$ - ions by charge exchange in two separate target cells instead of the single cell we currently use. We would achieve this by placing a small gas cell containing Xe just upstream of the $\mathrm{Li}$ vapor cell. The metastable $\mathrm{N}$ atoms produced in the first cell would then have a high probability of capturing a single electron in the second cell to produce the metastable states of the $\mathrm{N}$ anion.

We are also very interested in starting experiments that search for short lived excited states of negative ions. These transient states, which are extremely sensitive to electron correlation effects, manifest themselves as resonances in the photodetachment cross section in the vicinity of the opening of channels. Very little is known theoretically about such states in anions other than $\mathrm{H}$-. Such states are not easy to access since they require the use of a UV light source of narrow bandwidth. Our present flashlamped-pumped dye laser is not capable of reaching such short wavelengths. We have made a request to the University of Tennessee for the purchase of an excimer-pumped dye laser but at this time we do not know what the outcome will be. Our plans would be to try to access the even parity ${ }^{4 S}$ and ${ }^{4 D}$ excited states of $\mathrm{He}$. These states are optically coupled to the $4 \mathrm{P}$ ground state by a single UV photon. We propose to begin a collaboration with Dag Hanstorp from Chalmers University in Gothenburg, Sweden. At his institution there already exists both a small accelerator capable of producing beams of negative ions and state-of-the-art UV laser sources that would be ideal for the proposed experiments. The experiments at Chalmers would involve a search for excited states in the alkali metal anions and perhaps $\mathrm{He}^{-}$. 


\section{E. References}

[1]. D. J. Pegg, in The Physics of Electronic and Atornic Collisions, New York, 1989, edited by A Dalgarno, R. S. Freund, P. Kach, M. S. Lubell, and T. Lucatorto (American Inst. of Physics, New York, 1990 ), p. 233.

[2]. J. Dellwo, Y. Liu, D. J. Pegg, and G. D. Alton, Phys. Rev. A 45, 1544 (1992); J Dellwo, Y. Liu, C.Y. Tang, D.J. Pegg, and G.D. Alton, Phys. Rev. A 46, 3924 (1992).

[3]. Y. Liu, D.J. Pegg, J.S Thompson, J. Dellwo, and G.D. Alton, J. Phys. B 24, L1 (1991)

[4].A.W.Weiss, Phys.Rev.166,70(1968).

[J]. C.F.Bunge, M. Galen, R. Jauregui, and A. V. Bunge, Nucl. Instr. and Methods 202, 299 (1982); A. V. Bunge and C. F. Bunge, Phys. Rev. A 19, 452 (1979).[11].

[6]. D. R. Beck and C. A. Nicolaides, Int. J. Quantum Chem., 18, 467 (1984).

[7]. T. J. Kvale, G. D. Alton, R. N. Compton, D. J. Pegg, and J. S.

Thompson, Phys. Rev. Lett. 55, 484 (1985).

[8]. A. V. Bunge, Phys. Rev. A 33, 82 (1986).

[9]. C. E. Moore, Atomic Energy Leveis, Natl. Bur. Stand. (U.S.) Circ. No. 467 ( U.S GPO, Washington, D.C. ,1949), Vol 1.

[10]. W. R. Bozman, C. H. Corliss, W. F. Meggers, and R. E. Trees,

J. Research Nat. Bur. Std., 50, 131 (1953).

[11]. Y. K. Bae and J. R. Peterson, Phys. Rev. A 30, 2145 (1984). 
3. Report No: DOE/ER/13456-52

"Phorodetachment of the LI Negative Ion", J.Dellwo, Book of Abstracts of the Twelfth International Conference on the Applications of Accelerators in Research and Industry, Denton, TX,1992 
F. List of Publications: 1992-93

A. Journals and Published Proceedings

1. Report NO: DOE/ER/13456-48

" Photodetachment Cross Sections for Li-", J Dellwo, Y. Liu, C.Y. Tang, D.J.Pegg and G.D.Alton, Phys. Rev. A 46, 3924 (1992)

2. Report No: DOE/ER/13456-49

" An Investigation of Li- Photodetachment in the vicinity of the $22 P+\varepsilon s$ Threshold", J.Dellwo, Y.Liu, C.Y.Tang, D.J.Pegg and G.D.Alton, Nucl. Instr. and Methods ( to be published, 1993 ).

B. Conference and Workshop Abstracts

1. Report No: DOE/ER/13456-50

" Photodetachment Studies on Few-Electron Atomic Negative I Ions", D.J.Pegg, Book of Absracts, DOE Workshop on Atomic Physics, Ithaca, NY, 1992

2. Report NO: DOE/ER/13456-51

"Electron Affinity of Be", C.Y.Tang, D.J.Pegg, J.Dellwo and

G.D.Alton, Bull. Am. Phys. Soc., 37, 1681 (1992) 

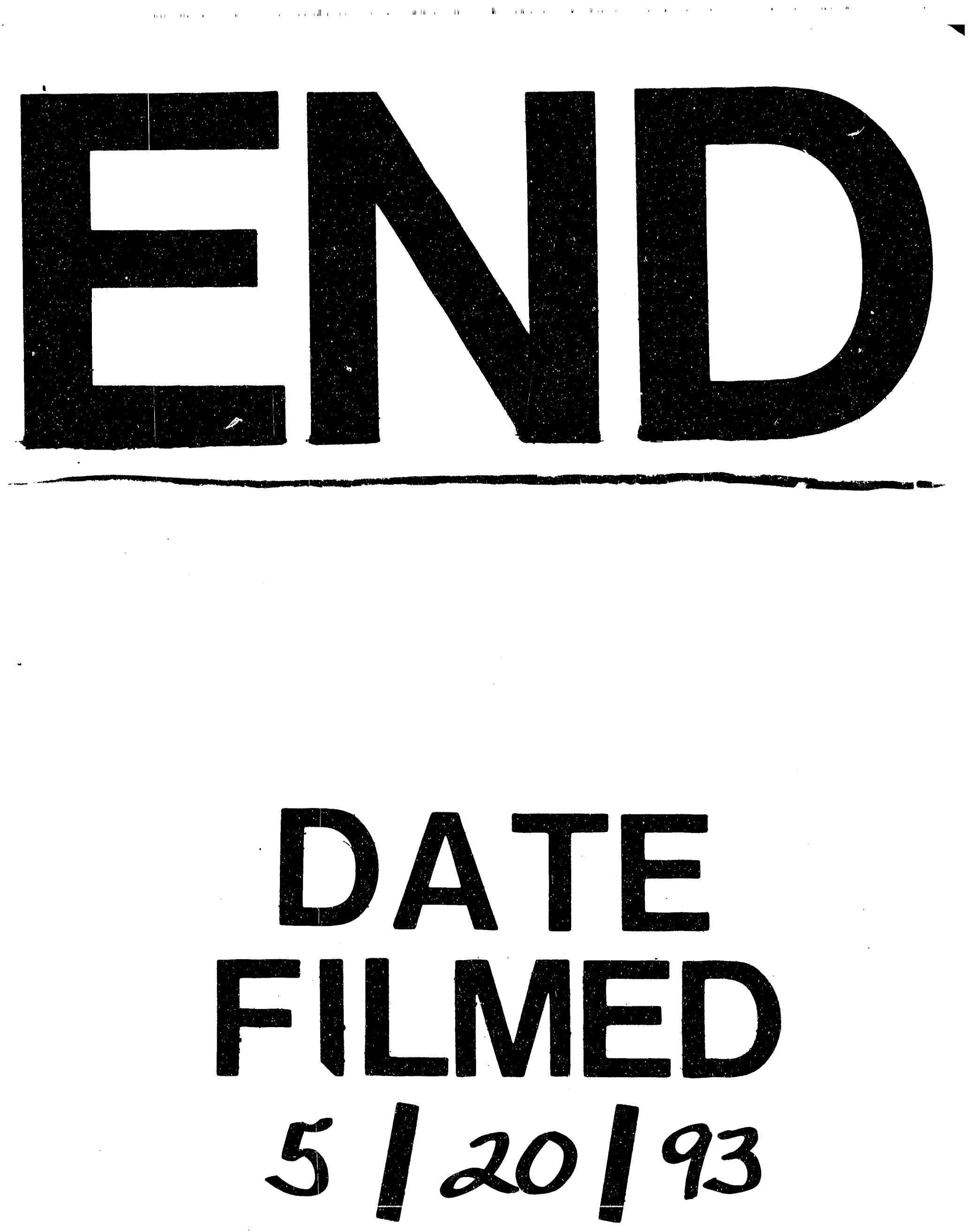
